\title{
Quasicrystal-Polymer Composites for Additive Manufacturing Technology
}

\author{
S. Kenzari ${ }^{a}$, D. Bonina ${ }^{a}$, A. Degiovanni ${ }^{b}$, J.M. Dubois $^{a}{ }^{*}$ And V. Fournée ${ }^{a}$ \\ ${ }^{a}$ Institut Jean Lamour (UMR 7198 CNRS Université de Lorraine), Parc de Saurupt, CS 50840, 54011 Nancy, France \\ ${ }^{b}$ Laboratoire d'Energétique et de Mécanique Théorique et Appliquée (UMR 7563 CNRS Université de Lorraine) \\ 2 Av. de la Forêt de Haye, TSA 6064, 54518 Vandoeuvre les Nancy, France \\ We present a new niche application of quasicrystalline powders that leads to a commercially successful de- \\ velopment of polymer-matrix composites. The process is based upon selective laser sintering, which is one of the \\ current most powerful additive manufacturing technologies in use in the mechanical industries. Characteristics of \\ the materials produced, such as porosity, friction and wear against hard steel, are evaluated and compared to the \\ state of the art. Insight into the production of metal-matrix composites, using a variant of the technology, is also \\ given. \\ DOI: 10.12693 /APhysPolA.126.449 \\ PACS: 81.05.Bx, 81.05.Qk, 81.05.Zx, 81.20.Ev, 83.80.Ab, 89.20.Bb
}

\section{Introduction}

Mechanical industries (automotive, aeronautics, etc.) are in increasing demand of net shaped products that are manufactured without waste of matter and are obtained directly at their final functional shape. New technologies have therefore been developed over the years with the view that the absence of shavings, compared to standard cutting and machining techniques, entails economical savings on various standpoints such as a reduction of the amount of material to be employed, the absence of storing and recycling wasted materials, and in the end a more friendly impact on the environment. Rapid prototyping is one such technology [1]. It decomposes any complex shape into a sequence of thin slices, which are cut out of flat material (steel, aluminium, plastic, etc.) and are later on assembled to reproduce the complex shape at the adequate scale by gluing, or screwing, or welding, etc. Optimisation of the slice cutting minimises the waste, but cannot eliminate it. Three-dimensional manufacturing technologies among which can be found additive layer manufacturing [2], and more appropriately in the present context, selective laser sintering (SLS for short hereafter) [3], provide more efficient technologies since they produce no waste at all and are capable of producing final-shape parts with respectable mechanical performances with no need of further binding between layers.

The purpose of this article is to present how quasicrystalline powders integrated in a polymer matrix enhance the flexibility of SLS for manufacturing complex mechanical parts while, in the mean time, they improve significantly some of the mechanical characteristics of those parts. The processing of the polymer-matrix composites is described in Sect. 2. Assessment of few major properties of those materials is the matter of Sect. 3. Extension

\footnotetext{
* corresponding author; e-mail:

jean-marie.dubois@univ-lorraine.fr
}

to the production of aluminium-matrix composites is explained in Sect. 4 before a conclusion comes in Sect. 5 .

\section{Processing of polymer-matrix composites}

The whole process can be divided in two basic steps, first atomization of the quasicrystalline powder, second manufacturing of the net-shape part by selective laser sintering. The most important issues of these two steps are described hereafter.

\subsection{Powder atomization}

Atomisation of quasicrystalline alloys was setup quite some time ago [4] when the industrial issue was the production of a new generation of frying pans coated with a layer of quasicrystal able to reduce adhesion of food on the one hand and offering better resistance to scratch on the other [5]. Gas atomization was used to this end, starting from molten alloy and ending in batches of spherical powder grains the size of which was distributed from sub-micronic sizes to about $100 \mu \mathrm{m}$ or more. Selecting an adequate range of powder diameters suitable for the envisaged application could be achieved by sieving after the atomisation step. Altogether, powders in the range 25$65 \mu \mathrm{m}$ were suitable for plasma torch spraying, the coating technology available at that time, whereas powders of smaller diameters appeared useful for the preparation of polymer matrix composites. Due to conflicting industrial strategies, the two application routes were unfortunately abandoned, even after the new frying pan came to the market and even after Sheares and Bloom had demonstrated the promises of polymer-matrix composites filled with quasicrystalline powders [6]. The know-how developed for atomisation was nevertheless transferred to an international materials producer ${ }^{\dagger}$, which is today able to sell batches of atomised powder of composition $\mathrm{Al}_{59} \mathrm{Cu}_{25} \mathrm{Fe}_{13} \mathrm{~B}_{3}$ (at.\%) that are sieved in a narrow range around $75 \mu \mathrm{m}$. Addition of few at.\% of boron lowers the melting temperature of the alloy and facilitates

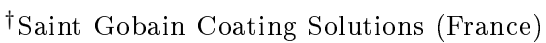


the atomisation process [7]. It leads to the formation of an Al-Fe boride, which improves the hardness of the material and makes it more wear resistant [8]. When brought to ambient atmosphere, the powder grains are covered by a layer of amorphous alumina the thickness of which is in the range of a fraction of a nanometre and evolves smoothly with increasing temperature below $500{ }^{\circ} \mathrm{C}[9]$. Yet, the phase content of the as-atomized grains is governed by the kinetics of the peritectic reaction that leads to the growth of the quasicrystalline phase from the CsCl-type $B 2$ phase that is stable at high temperature, and residual liquid [10]. Atomised powders are therefore multiphased and contain at least two phases, the quasicrystal (as the majority phase) and the cubic $\beta$-AlCuFe phase.

\subsection{Selective laser sintering}

We use an industrial device ${ }^{\ddagger}$ that allows the routine production of mechanical parts with dimensions of up to few tens of centimetres (Fig. 1). A bed of polymer mixed with quasicrystalline powder is prepared first. The polymer component is made of polyamine12 (PA hereafter), i.e. nylon particles ${ }^{\S}$ of diameter smaller than $75 \mu \mathrm{m}$ and amounts to $70 \%$ of the total volume. The flat surface of this bed is then illuminated, using a focused laser beam running on that surface under the control of a computer, thus drawing precisely a computer-assisted design of the part. The wavelength $(\lambda=10 \mu \mathrm{m})$ is selected in order to produce localised melting of the polymer $\left(T_{\text {melt }}=451 \mathrm{~K}\right)$, which is pre-heated in the bed at a temperature chosen under, but close to, its melting point $\left(T_{\text {bed }}=425-450 \mathrm{~K}\right)$. A new layer $(0.1 \mathrm{~mm}$ thickness $)$ is then added on top of the bed surface, which moved downwards by the same distance, and the process is continued layer by layer until the full volume of the part is obtained as a solid 3D body. The finished part can then be easily removed from the powder bed and the unused powder recycled for the next production step.

All kinds of solid volumes can be produced this way, which explains why SLS encounters such a brilliant success in industrial production, provided the final mechanical properties match the industrial demand. This is the reason why mechanical reinforcement of the polymer is an essential issue of SLS. Various reinforcement particles have been tried, including fibres, metals, or inorganic components (e.g. granite powder). In the following section, we will compare the properties of quasicrystal-reinforced nylon $(\mathrm{PA}+\mathrm{QC})$ with the properties of the unloaded material on the one hand, and with those of nylon-reinforced composites. One important issue is going to be the absence of porosity in $\mathrm{PA}+\mathrm{QC}$, whereas nylon as such, or being reinforced by various materials, shows an appreciable residual porosity which imposes a supplementary process to tighten the composite when

\footnotetext{
${ }^{\ddagger}$ Called a $S P r o{ }^{T M} 60 S L S{ }^{\circledR}$ Center by the profession.

$\S$ Supplied by $3 \mathrm{M}$ (USA).
}

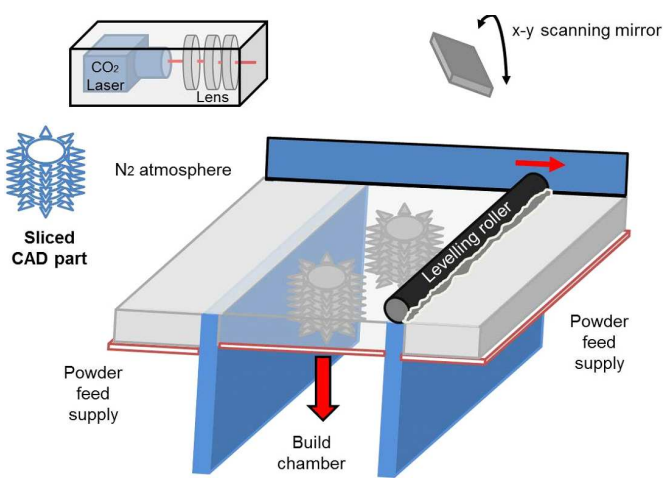

Fig. 1. Simplified sketch of the SLS device used in this study. The horizontal surface of a bed containing the blend of $\mathrm{QC}$ and nylon powders is prepared flat. It is then illuminated with an IR $\mathrm{CO}_{2}$-laser beam that traces layer by layer the shape of the part stored as a computer-assisted design file, which in turn monitors the beam. Any complex shape may be reproduced this way. The temperature of the blend is controlled close to the melting point of the polymer. A protective atmosphere is usually applied to protect the components from severe oxidation during processing.

used under pressure. This property of $\mathrm{PA}+\mathrm{QC}$ is unique and justifies the usage of quasicrystal powder. Its origin is related to the specific electronic properties of quasicrystals, owing to the absence of periodicity that cancels the Drude peak normally observed at low frequencies on the optical conductivity of a metal [11]. As a result, the absorption of infrared (IR) light is much higher in a quasicrystal compared to that in a metal like steel or an aluminium alloy. Simultaneously, emissivity is pretty low. Furthermore, heat conductivity is found to be low, compared to standard metals, at $T_{\text {bed }}$, which means that the powder grain will store the heat rise due to the IR irradiation long enough for the surrounding polymer to melt and fully wet it. Hence, it is possible to estimate the temperature rise of a powder grain during the time interval it is irradiated by the IR laser from the following equation:

$$
\begin{aligned}
\varphi & -h \pi D^{2}\left(T-T_{\text {bed }}\right)-\sigma \varepsilon \pi D^{2}\left(T^{4}-T_{\text {bed }}^{4}\right) \\
& =\frac{1}{6} \pi \rho C_{p} D^{3} \frac{\mathrm{d} T}{\mathrm{~d} t},
\end{aligned}
$$

where $\varphi=\frac{\alpha \varphi_{\mathrm{L}} D^{2}}{D_{\mathrm{L}}^{2}}$ is the energy brought into the particle by the laser beam, $\varphi_{\mathrm{L}}$ - the nominal power of the laser, $D_{\mathrm{L}}$ - the diameter of the laser beam and $D$ is that of the particle supposed of much smaller size, and $\alpha$ is its absorption coefficient at the laser wavelength. The other parameters are $h$ - the convection exchange coefficient at the QC-nylon interface, $\sigma$ - Stefan's constant, $\varepsilon-$ the emission coefficient of the QC grain, $\rho$ - its mass density and $C_{p}$ - its specific heat at constant pressure. The temperature increase $\Delta T$ is taken during the time interval $\Delta t$ of irradiation of the grain by the powder, which since $D \ll D_{\mathrm{L}}$ is simply given by $\Delta t=D_{\mathrm{L}} / v$ where $v$ is the drift velocity of the laser beam on the bed surface. 
Assuming on the one hand that the power distribution is homogeneous within a cross-section of the laser beam, and on the other hand neglecting the energy losses due to convection $(h \approx 0$ in the second, left side term of Eq. (1)) and to radiative emission ( $\varepsilon \approx 0$, third term), leads to the following first order approximation of the temperature rise:

$$
\Delta T \cong \frac{6}{\pi} \frac{\alpha}{\rho C_{p}} \frac{\varphi_{\mathrm{L}}}{D D_{\mathrm{L}}} v^{-1} .
$$

Considering numerical data for the physical properties of the $\mathrm{AlCuFeB}$ quasicrystal [7] leads to $\Delta T=42 \pm 5 \mathrm{~K}$, which represents a very significant temperature increase of the grain surface, able to melt the surrounding nylon. For comparison, in identical experimental conditions, a standard aluminium alloy would produce a temperature change of only $+5 \mathrm{~K}$ and stainless steel, a rise of $+1 \mathrm{~K}$. The molten polymer that surrounds the QC grain is therefore able to wet the grain in a far more efficient way than a metallic filler (characterized by $\alpha \approx 0$ ) would do, whereas its higher thermal conductivity would retain the temperature rise not long enough to achieve full wetting at its surface . The application niche we deal with in this article, although primarily concerned with mechanical properties, is therefore an indirect application of the specific electronic properties of quasicrystals.

\section{Properties and results}

Porosity, hardness, wear resistance, and friction coefficient, both against hard steel, were assessed in a comparative study against state of the art composites (non-filled nylon (PA) and nylon loaded with carbon and glass fibres or glass particles). The porosity was checked by producing leak-tight tests under air and water pressure up to 7 bars. Whereas all state-of-the-art composites showed significant leakage under these conditions, no leak, therefore no porosity, was observed for QC-reinforced composites. This result embodies an absolute advantage of our technology since it eliminates the post-processing impregnation treatment that is usually mandatory to obtain water or gas tight components using the other composites. Hardness was evaluated using a Shore D device, showing that filling with QC powder slightly increases the hardness to $79 \pm 1$ Shore units, instead of $74 \pm 2$ in conventionally filled composites. Traction tests however showed no difference regarding the ultimate tensile stress of both QC-filled and conventional materials.

Friction was studied using pin-on-disk tests performed at room temperature in ambient atmosphere with a spherical 100Cr6 hard steel indenter of $6 \mathrm{~mm}$ diameter. The normal load was set to $10 \mathrm{~N}$, the trace diameter to $10 \mathrm{~mm}$ and the velocity of the indenter relative to the polished surface of the composite to $16 \mathrm{~cm} / \mathrm{s}$. Optical

Taking into account radiative losses with an emission coefficient of 0.05 leads to a temperature increase $\Delta T=32 \mathrm{~K}$, which does not modify our conclusion. observation after ending the test was made to characterize the wear loss induced by friction during the test. Wear resistance of SLS disks was evaluated in water using a standard polishing technique ( $\mathrm{SiC}$ paper, 500 grit, normal load $10 \mathrm{~N}, 1 \mathrm{~min}$ ). Wear is expressed by volume loss. A bunch of results is shown in Fig. 2 regarding friction in the upper part of the figure and wear losses in the bottom part.

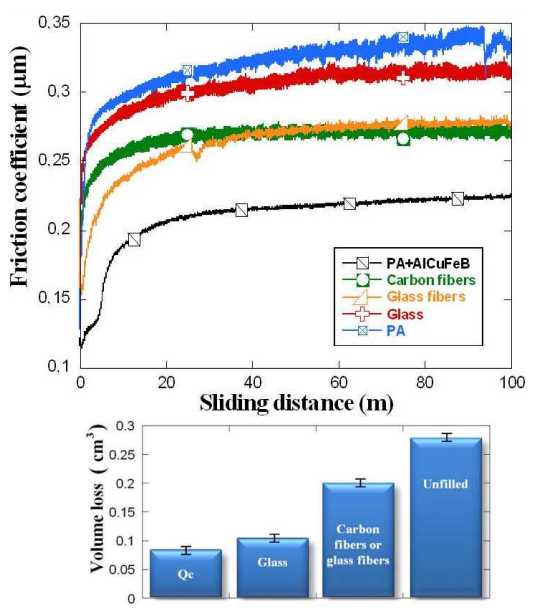

Fig. 2. Comparison between friction (top part) and wear (bottom part) data deduced from pin-on-disk tests against a spherical hard steel ball in ambient atmosphere (see text).

It turns out from this data that friction on a $\mathrm{PA}+\mathrm{QC}$ composite is about $75 \%$ of the figure observed on fibre-reinforced composites and half that observed on non-filled nylon or on nylon reinforced with glass particles. Simultaneously, wear is also reduced, but not in the same proportions as observed for friction. If the best solution is obviously provided by $\mathrm{PA}+\mathrm{QC}$, glass particle filling achieves an almost identical result whereas fibres and nylon alone give quite poorer results. Whatever the results of this comparative study, a clear advantage is brought by filling polyamide 12 with quasicrystalline powder. On the one hand, it simplifies the processing of polymer-matrix composites by SLS. On the other hand, remarkable friction and wear behaviour is detected for mechanical parts to be used in contact mechanics. A patent was filled to secure this invention [12].
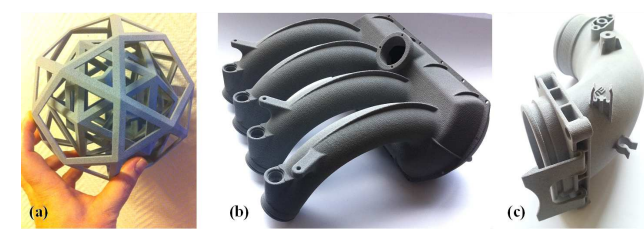

Fig. 3. Examples of parts produced by SLS using polyamide12 filled with quasicrystalline powder as described in text. The part in (a) is self-understood by experts from the ICQ community. In (b) and (c) there are shown real parts actually used by a French car producer. The size of the exhaust component shown in (b) is typically $20 \times 30 \times 40 \mathrm{~cm}^{3}$. 
The invention has now passed the initial steps of intellectual property rights [13]. Marketing of products derived from this technology is now under way by a small and medium size enterprise ${ }^{\dagger \dagger}$ located close to our laboratory. Figure 3 presents three examples of products manufactured and sold by this company under trademark $\mathrm{PAQc}^{\circledR}$.

\section{Aluminium-matrix composites}

The same methodology may be applied to the production of metal-matrix composites reinforced with QC powders as was first imagined by Tsai et al. [14]. In our method however [15], we start from a polymer-matrix composite prepared by SLS according to the method described in previous sections, in such a way that it is solid enough to be handled with no damage. Then, the preform obtained this way is placed on a sacrificial tab and heated under primary vacuum to evaporate the polymer. A filler consisting of an aluminium alloy of melting point below the one of the quasicrystal is placed on the sacrificial tab. It penetrates by capillarity in the pores left open by the polymer, thus binding the quasicrystal grains together (Fig. 4). Phase transformations, according to the

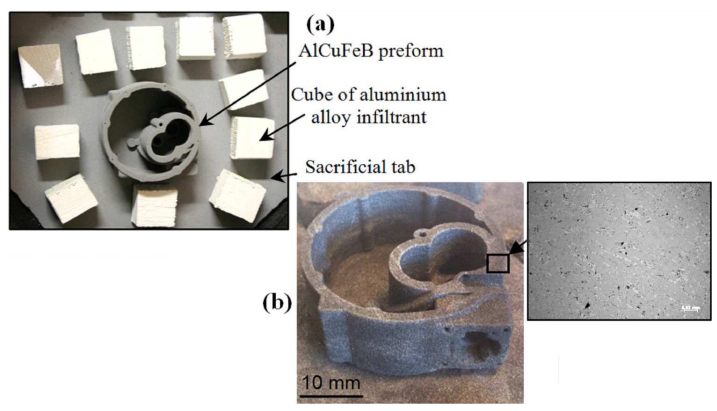

Fig. 4. (a) View from the top of the crucible containing the $\mathrm{AlCuFeB}$ based preform and small cubes of $\mathrm{Al}$ alloy infiltrant placed on a sacrificial tab. (b) The corresponding part and optical micrograph after infiltration with an Al-1050 alloy. The surface of the part was prepared by standard mechanical polishing before observation. It shows little residual porosity and confirms the actual obtention of a novel metal-matrix composite reinforced by QC particles.

equilibrium phase diagram, take place at the QC-alloy interface and participate in the composite strength. Relatively complex components can be produced this way at an acceptable industrial cost. This new method is still under test before commercial development can start.

\section{Conclusion}

We have presented a novel application niche for quasicrystalline powders. It is based upon selective laser sintering, one of the promising new additive manufacturing technologies nowadays in full industrial expan-

\footnotetext{
††Ateliers CINI, www.cini.fr, bd. Tolstoï, BP 17, F-54510 Tomblaine.
}

sion. Polymer-matrix composites reinforced with quasicrystalline particles show definite advantage in comparison to the state of the art. They have already reached the market of automotive components. An alternative technology that would allow production of light-weight metal-matrix composites, but is still under development, was introduced as well. Both technologies capitalize on the efforts dedicated two decades ago to gas atomization of quasicrystalline alloys.

\section{Acknowledgments}

We are most grateful towards Ateliers CINI for financing one of us (S.K.) over the years. Funding by Conseil Régional de Lorraine is also acknowledged.

\section{References}

[1] C. Barlier, Method for making mechanical parts by decomposition into layers, US Patent 7097729 (2004).

[2] J.C. André, A. Le Mehauté, O. De Witte, Device for producing a model of an industrial part, French Patent FR 2567668 (1984).

[3] C. Deckard, J. Beaman, J. Darrah, Method and apparatus for producing parts by selective sintering, Patent WO 9208567 (1988).

[4] J.M. Dubois, A. Proner, B. Bucaille, Ph. Cathonnet, C. Dong, V. Richard, A. Pianelli, Y. Massiani, S. Ait-Yaazza, E. Belin-Ferré, Ann. Chim. Fr. 19, 3 (1994).

[5] J.M. Dubois, P. Weinland, Coating materials for metal alloys and metals, European Patent 0356287 A1 (1990).

[6] V.V. Sheares, P.D. Bloom, Quasicrystalpolymer composite materials and methods, Patent WO 005,653,8 (2000).

[7] J.M. Dubois, P. Archambault, B. Colleret, Quasicrystalline aluminium heat protection element and thermal spray method to form elements, US Patent 5888661 (1999).

[8] V. Brien, V. Khare, F. Herbst, P. Weisbecker, J.B. Ledeuil, M.C. De Weerd, J.M. Dubois, J. Mater. Res. 19-10, 2974 (2004).

[9] P. Weisbecker, G. Bonhomme, A. Cael, L. Zhang, J.M. Dubois, Mater. Res. Soc. Symp. Proc. 643, K9.2.1 (2001).

[10] C. Dong, J.M. Dubois, M. de Boissieu, Ch. Janot, J. Phys. C, Condens. Matter 2, 6339 (1990).

[11] V. Demange, A. Milandri, M.C. de Weerd, F. Machizaud, G. Jeandel, J.M. Dubois, Phys. Rev. B 65, 144205 (2002).

[12] S. Kenzari, V. Fournée, Method for producing a part including aluminium, French Patent FR 2950826 (2009), PCT WO2011/039469 (2011).

[13] S. Kenzari, D. Bonina, J.M. Dubois, V. Fournée, Mater. Des. 35, 691 (2012).

[14] A.P. Tsai, K. Aoki, A. Inoue, T. Masumoto, J. Mater. Res. 8-1, 5 (1993).

[15] S. Kenzari, V. Fournée, Method for producing aluminium alloy parts, French Patent FR 2929541 (2008), PCT WO 2009/144405 (2009). 